\title{
Graceful exit from a stringy landscape via MSSM inflation
}

\author{
Rouzbeh Allahverdi* \\ Perimeter Institute for Theoretical Physics, Waterloo, Ontario, N2L 2Y5, Canada \\ Andrew R. Frey ${ }^{\dagger}$ \\ Physics Department, McGill University, Montréal, Quebec, H3A 2T8, Canada
}

Anupam Mazumdar

NORDITA, Blegdamsvej-17, Copenhagen-2100, Denmark

(Received 4 May 2007; published 2 July 2007)

\begin{abstract}
The cosmological evolution of the string landscape is expected to consist of multiple stages of old inflation with a large cosmological constant, each of which ends by tunneling. Old inflation has a wellknown graceful exit problem as the observable universe becomes empty, devoid of any entropy. Simultaneously, in the quest for reheating the right degrees of freedom, it is important that the final stage of inflation reheat the standard model sector. It is known that inflation can occur naturally along a flat direction of the minimal supersymmetric standard model (MSSM), solving the reheating problem, but the initial conditions require a large degree of fine-tuning. In this paper, we study how inflation of a MSSM flat direction can be embedded into the string theory landscape to solve both the graceful exit problem of old inflation and the fine-tuning problem of MSSM inflation, elaborating on ideas of Bousso and Polchinski. The fluctuations of the MSSM flat direction during old inflation create regions with initial conditions favorable for eternal inflation, which also allows the cosmological constant to continue to relax. This final phase of inflation also provides all the usual benefits of MSSM inflation, including straightforward reheating into standard model degrees of freedom.
\end{abstract}

DOI: 10.1103/PhysRevD.76.026001

PACS numbers: 11.25.Wx, 12.60.Jv, 98.80.Cq

\section{INTRODUCTION}

We expect that the early universe, prior to inflation, existed in a state of high energy density, in particular, near the cutoff scale of the 4D effective field theory we know today. In string theory, this cutoff is typically expected to be near the fundamental string scale. Also, there is some evidence that string theory has a "landscape" of metastable vacua with a varying cosmological constant, moduli vacuum expectation values (VEVs) (and therefore couplings), a supersymmetry (SUSY) breaking scale, and so on, which can be studied using statistical arguments (see [1-3] for reviews).

Since many of these metastable vacua have large energy densities (as we describe below), it seems likely that the early universe could have existed as a de Sitter spacetime with a large cosmological constant, which then decayed by tunneling, as in old inflation [4]. In fact, such a cosmology with multiple stages of inflation [5] provides a mechanism by which the full landscape of vacua is populated, as in [68]. With some caveats, this mechanism can also relax the cosmological constant quickly [6-10]. In particular, the cosmological constant must be able to decay even though other sectors will dominate once the energy density reaches $\sim 1(\mathrm{TeV})^{4}$.

\footnotetext{
*rallahverdi@perimeterinstitute.ca

†rey@hep.physics.mcgill.ca

‡anupamm@nordita.dk
}

One obvious worry about this picture is that, if the universe were to tunnel out of the false vacuum, then the universe would be devoid of any entropy as the nucleated bubble would keep expanding forever with a negative spatial curvature. Such a universe would have no place in a real world, so it is important that the last stage of inflation be driven by a slow-roll phase that must explain the following:

(i) The observed temperature anisotropy in the cosmic microwave background radiation and the subtle observational details [11]. This implies that the last tunneling event must occur at least 60 e-foldings before the end of inflation.

(ii) Reheating into standard model (SM) like degrees of freedom, so that baryons and cold dark matter can be produced.

(iii) To solve the cosmological constant problem, the slow-roll phase must allow the population of vacua with smaller cosmological constants, which is facilitated by eternal inflation.

The first criterion has been addressed by [7], and we will see that the minimal supersymmetric standard model (MSSM) can facilitate all the criteria.

Also, it was recently realized that the MSSM has all the necessary ingredients for successful inflation, whose parameters can possibly be tested at the CERN LHC [12-15]. Moreover, this inflation has a graceful exit which creates conditions for producing baryons and cold dark matter. The inflaton is a gauge invariant MSSM flat direction (for a review on MSSM flat directions, see $[16,17])$. There are 
potentially two candidates, $L L e$ and $u d d$, where the superfield $L$ stands for an $S U(2)_{L}$ lepton doublet, while $e, u, d$ are superfields standing for the right-handed components of the electron and up- and down-type quarks. Both the inflaton candidates carry SM lepton and baryon numbers. After inflation, the inflaton decay directly creates baryons, leptons, and cold dark matter (the lightest superpartner is a candidate for cold dark matter [18]). ${ }^{1}$ This decay addresses the second point above.

Inflation within the MSSM occurs near a saddle point of the flat direction potential, which is lifted by the soft SUSY breaking mass term, the nonrenormalizable superpotential, a potential supergravity mass correction, and the $A$-term [22,23]. All these contributions play an important role. Although none of the individual terms would give rise to inflation [24], all the terms combined provide a unique saddle point condition in the gravity mediated SUSY breaking case [12-14] and in the gauge mediated SUSY breaking case [15]. In these cases, the first nontrivial derivative of the inflaton potential is the third derivative, which alone determines the dynamics. This MSSM inflation matches the current WMAP observations with the right amplitude and the spectral tilt $[14,25,26]$. One important result is that the MSSM inflation produces many efoldings of slow-roll inflation, $\mathcal{N}_{e} \sim 10^{3}$, with a preceding self-reproduction regime. Therefore, MSSM inflation can satisfy the first and third criteria above. One difficulty of MSSM inflation, though, is that it requires a $10^{9}$ finetuning of the initial conditions for slow roll (and $10^{13}$ for self-reproduction) and a $10^{9}$ fine-tuning of the $A$-term $[27,28]$.

As we shall see, old inflation on the landscape and MSSM inflation complement each other nicely. The landscape picture suggests that the $A$-term fine-tuning is no longer a problem. Also, as in [7], fluctuations of the MSSM inflaton during false vacuum inflation naturally fine-tune the initial conditions for MSSM inflation, i.e. why the MSSM inflation occurs near the saddle point of the potential $[12,14,29] .{ }^{2}$ On the flip side, MSSM inflation provides a period of eternal inflation followed by many e-foldings of slow-roll inflation, which allows tunneling to solve the cosmological constant problem and further creates the necessary temperature anisotropies. Finally, MSSM infla-

\footnotetext{
${ }^{1}$ Often the inflaton is treated as an absolute gauge singlet [19]. Even string inspired models, the inflaton is an absolute SM gauge singlet. In all these cases reheating into the SM-like degrees of freedom is not so obvious and is plagued with the uncertainty due to the inflaton coupling to the matter field [20]. As an absolute gauge singlet the inflaton can have any coupling strength to the SM degrees of freedom; there is no symmetry argument which constrains the coupling [21].

${ }^{2}$ This would be difficult to explain otherwise. For example, one may not invoke thermal effects to trap the flat direction near the saddle point as it does not correspond to a false minimum, or a point of enhanced symmetry.
}

tion, by its nature, allows simple reheating into the SM sector.

We will begin our discussion with a broad brush-stroke review of the string landscape and the SM-like sector of it, first showing why we expect that the landscape can accomplish fine-tuning of the $A$-term. Then we discuss the time scales associated with tunneling events on the landscape, showing why eternal inflation seems necessary for the cosmological constant problem and why the arguments of [7] require a slight extension. We will also show why we can assume the MSSM to be present throughout the chain of tunneling events. Following our discussion of the MSSM in the landscape, we give a brief review of MSSM inflation. Then we explain how old inflation on the landscape can set the initial conditions for eternal inflation in the MSSM sector, using an improved form of the arguments of [7]. In short, the MSSM flat directions, being light, obtain random quantum jumps, which displace them to large expectation values [19]. Alternately, supergravity corrections to the MSSM can trap the flat directions in a false minimum (as in [30-32]).

\section{OLD INFLATION ON THE LANDSCAPE}

There are three main points we wish to make clear in our review of the landscape picture of string theory vacua. First, we wish to explain the current picture of landscape, including large (string-scale) cosmological constants, especially reviewing the distribution of SM-like metastable vacua on the landscape. We will also review the scale of SUSY breaking and corrections to the MSSM Lagrangian due to the landscape sector and argue that the $A$-term can scan over the necessary range for MSSM inflation. Second, we will discuss tunneling rates for the decay of each false vacuum, describing the phase of old inflation prior to MSSM inflation. With these rates in hand, we will see why the original solution of [7] for the empty universe problem is not quite complete. Finally, we will see why eternal inflation is necessary for tunneling on the landscape to solve the cosmological constant problem in a realistic cosmology.

\section{A. Landscape with large $\Lambda$}

The most basic fact about the landscape (which is usually not discussed, being of little interest for present-day physics) is that the cosmological constant is generically large. A simple way to see this follows [7], which describes the landscape contribution to the cosmological constant as arising from string theory flux. In this picture, the vacuum energy

$$
V=M_{P}^{2} \Lambda=M_{P}^{2} \Lambda_{0}+\alpha^{\prime-2} \sum_{i} c_{i} n_{i}^{2},
$$

where $c_{i} \lesssim 1$ are constants and $n_{i}$ are flux quantum numbers (note that $\Lambda$ has dimension mass ${ }^{2}$ in our notation as it enters the Einstein equation as $\Lambda g_{\mu \nu}$ ). It is clear that a large 
$\Lambda$ corresponds to a large radius shell in the space of flux quanta, so a larger $\Lambda$ will have more possible states. All in all, string theory (from the landscape point of view) could have from $10^{500}$ to even $10^{1000}$ vacua [1-3,7], with the vast majority of those having large cosmological constants.

In addition, our knowledge of the distribution of gauge groups over the landscape suggests that 1 out of $10^{10}$ vacua have the SM spectrum, at least in simple models [33-36]. Even if this fraction is much smaller on the whole landscape, there are so many vacua that it seems likely very many will have a SM-like spectrum. For simplicity's sake, we will consider only this SM-like subset of the landscape in the rest of the paper, meaning we are ignoring possible transitions among vacua with different gauge groups in our cosmological model. We will justify this assumption in Sec. II C.

Given that the cosmological constant is large, at what scale is supersymmetry in the SM sector? We need to know this because we will want to follow the behavior of MSSM degrees of freedom throughout our cosmology, including time spent in vacua of large $\Lambda$, and we need to estimate corrections to the MSSM action. From statistical arguments (again, see [1-3] for more references), most vacua should have badly broken supersymmetry, with large $F$-terms in the supersymmetry breaking sector. ${ }^{3}$ However, we assume that these $F$-terms are not in the SM sector, which can therefore be described as the MSSM (though perhaps with large soft breaking terms). In addition, there exist vacua with a large cosmological constant that are "almost supersymmetric" in the sense of [2]. These vacua have vanishing (or nearly vanishing) $F$-terms, and their cosmological constant and supersymmetry breaking are provided by a $D$-term, such as that created by an antibrane. Upon decay of the $D$-term, the remaining vacuum has a small $\Lambda$ and low energy supersymmetry. The original model of [37] is almost supersymmetric in this sense because anti-D3-branes provide both the cosmological constant and supersymmetry breaking. We can also consider the toy "friendly landscape" models of [38], in which the dynamics of $N$ scalars create a landscape of vacua. In the supersymmetric case,

$$
M_{P}^{2} \Lambda=M_{\mathrm{sb}}^{4}-\frac{3|W|^{2}}{M_{P}^{2}},
$$

where $M_{\mathrm{sb}}$ is the supersymmetry breaking scale of a hidden sector (not the $N$ scalars) and the superpotential falls in the range

$$
0 \leq|W|^{2} \leq \sqrt{N} M_{\star}^{6}
$$

with $M_{\star}$ the cutoff.

In any of these cases, though, the scale of supersymmetry breaking in the MSSM sector is controlled in the early universe by the form of mediation. If supersymmetry

\footnotetext{
${ }^{3}$ This holds true even with small $\Lambda$.
}

breaking is gravity mediated, then the soft masses for the MSSM can be quite small, $m^{2} \sim M_{\mathrm{sb}}^{4} / M_{P}^{2}$, while the vacuum energy ranges over the landscape from zero (or potentially negative values) up to $M_{\mathrm{sb}}^{4}$. Even the very nonsupersymmetric vacua with large $\Lambda$ might be viable for our purposes. Suppose that the MSSM feels supersymmetry breaking from both a landscape sector and a hidden sector (the hidden sector provides the present-day SUSY breaking), with gravity mediation from the landscape sector. At large $\Lambda$, we find that the corrections to the MSSM soft masses from the landscape factor are $m \sim \Lambda^{1 / 2} \sim H$, so these corrections are the same as the Hubble corrections of [30-32]. We will consider these corrections explicitly in Sec. III.

We also want to know whether we can expect the $A$ parameter of the MSSM to vary over different values on the landscape in order to carry out the fine-tuning of one part in $10^{9}$ necessary for MSSM inflation. As it turns out, the $A$-term breaks $U(1)$ symmetry of the flat direction in the $\mathrm{MSSM}^{4}$ so the landscape should scan values of $A / M_{P}$ (the appropriate dimensionless combination) around $A=0$ in the sense of [38]. In this case, we need a value of $A \sim$ $10^{-16} M_{P}$ with a tuning of $\delta A / A \sim 10^{-9}$, so we need at least $10^{25}$ MSSM vacua to achieve the right vacuum spacing. Given the large number of MSSM vacua that seem likely to exist, it seems a safe assumption that the landscape can accomplish the necessary tuning of $A$. Again, most of these SM-like or MSSM-like vacua will have large $\Lambda$, so it seems reasonable to think that a generic initial condition for cosmology is one of these large $\Lambda$ vacua.

\section{B. Decay time scales and inflation}

The decay rate per volume (by tunneling) of a metastable vacuum to the nearest neighboring vacuum ${ }^{5}$ takes the form

$$
\Gamma / V=C \exp \left(-\Delta S_{E}\right)
$$

where $C$ is a one-loop determinant and $\Delta S_{E}$ is the difference in Euclidean actions between the instanton and the background with a larger cosmological constant. The determinant $C$ can be at most $C \lesssim M_{P}^{4}$, simply because $M_{P}$ is the largest scale available, and estimates (ignoring metric fluctuations) give a value as small as $C \sim r^{-4}$, with $r$ the instanton bubble radius [41]. If we therefore look at a decay rate in a (comoving) Hubble volume, we find

\footnotetext{
${ }^{4}$ This can simply be an $\mathrm{R}$ symmetry that multiplies the flat direction by a phase, which we can require the MSSM Lagrangian to respect before adding $A$-terms.

${ }^{5}$ To avoid subtleties, we require that both states have nonnegative energy density. See, for example, [39] for issues in the negative $\Lambda$ case. Also, [40] has discussed the importance of negative $\Lambda$ vacua in possibly separating parts of the landscape from each other. We adopt the view, as discussed in that paper, that the landscape is sufficiently complicated that there are no isolated regions.
} 


$$
\Gamma \lesssim \frac{M_{P}^{4}}{H^{3}} \exp \left(-\Delta S_{E}\right) .
$$

Especially with a large Hubble scale, the associated decay time is much longer than $1 / H$, given that typically $\Delta S_{E} \gg$ 1.

In fact, as given in [42] following [43-45], the Euclidean action takes the form

$$
\Delta S_{E}=2 \pi^{2} r^{3} \tau_{e}
$$

where the bubble radius and effective tension $\tau_{e}$ are given in terms of dimensionless cosmological constants $\lambda=$ $M_{P}^{4} \Lambda / \tau^{2}$, and the actual tension of the bubble wall $\tau$. The full formulas are listed in the Appendix.

Unfortunately, a given tunneling process in the landscape probably has $\lambda_{+} \sim 1$ and $\lambda_{-} \lesssim 1$. However, it may be instructive to look at three limits, $\lambda_{+} \rightarrow 0, \infty$. In the latter case, we simultaneously take $\lambda_{-} \rightarrow 0$ or $\lambda_{-} \rightarrow \lambda_{+}$ (these are the same for $\lambda_{+} \rightarrow 0$ ). As $\lambda_{+}$vanishes, we find

$$
\Delta S_{E} \rightarrow 24 \pi^{2} \frac{M_{P}^{6}}{\tau^{2} \lambda_{+}}=24 \pi^{2} \frac{M_{P}^{2}}{\Lambda_{+}} .
$$

(The tension surprisingly drops out.) For $\lambda_{+} \rightarrow \infty$, we find the limits

$$
\begin{gathered}
\Delta S_{E} \rightarrow 12 \pi^{2} \frac{M_{P}^{6}}{\tau^{2} \lambda_{+}}=12 \pi^{2} \frac{M_{P}^{2}}{\Lambda_{+}} \quad\left(\lambda_{-} \rightarrow 0\right), \\
\Delta S_{E} \rightarrow 6 \sqrt{3} \pi^{2} \frac{M_{P}^{6}}{\tau^{2} \lambda_{+}^{3 / 2}}=6 \sqrt{3} \pi^{2} \frac{\tau}{\Lambda_{+}^{3 / 2}} \quad\left(\lambda_{-} \rightarrow \lambda_{+}\right) .
\end{gathered}
$$

The only parametrically different behavior is the last limit as $\lambda_{ \pm} \rightarrow \infty$, which either means that the tension is very small or that the cosmological constants are both very large (or that $M_{P} \rightarrow \infty$, corresponding to field theory without gravity). Technically, the instanton approximation can break down in the limit (9), if the Euclidean action becomes much smaller than 1 (as a full quantum mechanical treatment would be necessary), but the important point is that $\Gamma / H \gtrsim 1$ if that is the case. From (5), we might expect that $\Gamma / H \gg 1$, but we should remember that the coefficient of the exponential in (5) is an upper limit and that quantum mechanical effects could limit the decay rate to $\Gamma \sim H$.

Therefore, we expect small jumps in $\Lambda$ with small tension $\tau$ to be the most common decays, which can in fact be quite rapid. Recently, [10,46,47] have argued that a series of decays in this last limit can give a novel form of inflation (dubbed "chain inflation") in which each metastable vacuum gives less than an e-folding, but which can overall have many e-foldings. ${ }^{6}$ In particular, in

\footnotetext{
${ }^{6}$ Recently, [48] discussed the set of vacua in a particular region of the landscape, and this "topography" may be relevant for discussions of chain inflation.
}

a large volume flux compactification model (ignoring warping) like those of [7], there is a range of flux quanta such that $\Delta S_{E} \ll 1$ (and $\Lambda<M_{P}^{2}$ ), so $\Gamma / H \sim 1$. In a warped flux compactification, the limit (9) can still apply, but, using the logic of $[20,42,49]$, the tunneling rate is somewhat slower, but similar (see the Appendix for details of these calculations). However, in the landscape, chain inflation does not have an a priori graceful exit [10]; the argument of [7] does not quite apply because it requires a large change in cosmological constant in the final decay before low-scale inflation (without inflaton trapping). In Sec. III, we give an improved form of the argument of [7] that shows how old inflation on the landscape can source MSSM inflation without inflaton trapping and with only small jumps in the cosmological constant.

Note that, when MSSM inflation starts, the "bare" cosmological constant (that not associated with the MSSM inflaton) might still be considerably larger than the present value. This means that further instanton decays should take place to reduce the bare cosmological constant, and these decays should occur during MSSM inflation in order for the bubble regions to grow long enough. Since it seems likely that the instanton bubble tension will be large compared to the scale of MSSM inflation, the decay rate will be given by (7), which is highly suppressed. This would then require MSSM inflation to last for extremely many e-foldings. Fortunately, MSSM inflation naturally includes a self-reproduction (eternal inflation) regime prior to slow roll [12-14]. In other words, it seems that a lowscale eternal inflation is a necessary part of using decays to solve the cosmological constant problem, and MSSM inflation includes it naturally. Eternal inflation solves several of the problems relating false vacuum inflation to observations, and it is particularly viable at the $\mathrm{TeV}$ scale of MSSM inflation [50,51].

We have two more comments regarding additional types of transitions on the landscape. First, [9] argues that resonance can play an important role in tunneling across a landscape of many metastable vacua; we would not find qualitative differences in that case, but decays could occur more quickly. Second, we have discussed thin-wall instantons, as were first studied in [43-45]. However, in some cases of very large tension, so-called Hawking-Moss instantons [52] can dominate the decay rate. This typically occurs when the potential barrier separating two vacua is very wide, but, unless the barrier is also very shallow, the decay rate is approximately given by (7). Because the potential barriers are typically expected to be quite tall on the landscape (up to string-scale energy densities), we will not treat Hawking-Moss instantons separately. Note also that there has been some recent discussion in the literature on the physical meaning of the Hawking-Moss instanton (e.g. [53,54]), but we will not delve into those subtleties here. 


\section{Following all paths}

As has been emphasized by $[7,8]$, the whole landscape, including the MSSM-like vacua, will be populated eventually almost independently of initial conditions due to repeated transitions among the different states on the landscape. In fact, if we ignore sinks in the landscape, eventually all the states will be populated with a fraction of the comoving volume given by a thermal distribution (see $[40,55,56]$ for a recent discussion including sinks).

What is important for us is that, because transitions are possible to states with a higher cosmological constant, each bubble of a given state will contain bubbles of every other state. This fact means that any given path through the vacua of the landscape will be followed by some region of comoving volume. In particular, we can consider a history in which some patch of comoving coordinates enters a state with a MSSM sector with the appropriate tuning of the $A$-term at a high cosmological constant and then proceeds to decay by reducing the cosmological constant without affecting the MSSM sector in any significant way. Therefore, we are justified in assuming that we can follow the behavior of MSSM fields and their actions throughout the process of old inflation on the landscape. Incidentally, these considerations are necessary for any application of the arguments of [7] for the exit from old inflation; the low energy inflaton must exist in the states with a larger cosmological constant.

\section{MSSM INFLATION}

Let us now discuss what happens to the observable sector in the background of inflationary spacetime. As we have noticed, the universe cascades from a large cosmological constant to another somewhat smaller cosmological constant as time progresses. Given the decay time, it is fairly evident that a huge number of e-foldings can be generated.

However, in order to have a sensible phenomenology, it is important that the last phase of inflation is primarily driven by MSSM-like vacua. Furthermore, it is equally pertinent that there exists a graceful exit of inflation within the MSSM. This is possible provided there is no potential barrier in the MSSM-like sector. Otherwise, the universe would nucleate in a cold state with a negative spatial curvature, because the bubble collision rate would not overcome the expansion rate [4]. In this regard, slow-roll inflation in the MSSM sector as discussed in [12-15] is ideal. We now ask how the dynamics of the MSSM flat direction will unfold during the era of false vacuum inflation.

During the false vacuum inflation the energy density of the universe, and hence the expansion rate $H_{\text {false }}$, remains constant for a given $\Lambda$. The flat direction potential receives corrections from the soft SUSY breaking mass term; the nonrenormalizable superpotential $W \sim \lambda \Phi^{n} / M_{P}^{n-3}$, where $W$ is the superpotential, $\Phi$ denotes the flat direction, and $\lambda_{n}$ is an $\mathcal{O}(1)$ coefficient $^{7}$ (we also assume that the cutoff is given by the 4-dimensional Planck mass) ${ }^{8}$; and corrections due to the large Hubble expansion for a minimal choice of Kähler potential. These corrections, parametrized by two constants $c \sim \mathcal{O}(1)$ and $a \sim \mathcal{O}(1)$, result in $[16,17,22,23]^{9}$

$$
\begin{aligned}
V= & \frac{1}{2}\left(m_{\phi}^{2}+c H_{\text {false }}^{2}\right)|\phi|^{2}+\left[\left(A+a H_{\text {false }}\right) \lambda_{n} \frac{\phi^{n}}{n M_{P}^{n-3}}+\text { H.c. }\right] \\
& +\lambda_{n}^{2} \frac{|\phi|^{2(n-1)}}{M_{P}^{2(n-3)}},
\end{aligned}
$$

where the soft SUSY breaking mass term is generically small compared to the Hubble expansion rate of the false vacuum, $m_{\phi} \sim 1 \mathrm{TeV} \ll H_{\text {false }}$. We define $\Phi \equiv \phi e^{i n \theta}$, and the dynamics of $\phi$ in an inflationary background depends on $c$ and $a$. Therefore we consider different cases separately.

It turns out that the dynamics of the MSSM flat direction largely follow the physics discussed in [7]. We review and elaborate on their discussion in the context of the MSSM and give an important improvement on their result in the context of negligible Hubble corrections.

\section{A. Positive Hubble induced corrections}

A positive Hubble induced correction provides $c \sim$ $+\mathcal{O}(1), a \sim \mathcal{O}(1)$. This is a typical scenario when the Kähler potential for the string modulus comes with a canonical kinetic term. Although this could be treated as a special point on the Kähler manifold, it is nevertheless important to discuss this situation. This is also the simplest scenario out of all possibilities. A generic flat direction gets a large mass of the order of the Hubble expansion rate; therefore, its fluctuations are unable to displace the flat direction from its global minimum.

On phenomenological grounds, this is an uninteresting and undesirable case, since the bubble does not excite any of the MSSM fields. Therefore, the bubble remains empty and devoid of energy with no graceful exit of inflation from the false vacuum. The universe continues cascading to smaller $\Lambda$ with smaller $H_{\text {false }}$. Eventually, when $H_{\text {false }} \leq$ $m_{\phi}$, the MSSM flat directions would be free to move.

\footnotetext{
${ }^{7} \lambda_{n}$ might actually be larger or smaller; for example, the cutoff might be given at a smaller scale (such as a Kaluza-Klein scale) or $\lambda_{n}$ might be suppressed as in [57]. However, $\lambda_{n}$ changes only the VEV of $\Phi$ and not the physics of inflation, so we will ignore this point.

${ }^{8}$ The nonrenormalizable term can be barred if the flat direction under consideration preserves R symmetry; otherwise, it is lifted by higher order superpotential operators (see $[22,23,58]$ ).

${ }^{9}$ The origin of the Hubble induced terms is due to couplings between the modulus which drives the false vacuum inflation and the MSSM sector. A priori, even in string theory, the Kähler potential for the modulus is not well known at all points of the parameter space. Similarly, within the MSSM, the Kähler potential is unknown. Therefore, the coefficients $c, a$ are not fixed. For a no-scale-type model, the Hubble induced corrections are vanishing at tree level; however, they do appear at one loop, $|c| \sim 10^{-2}[59,60]$.
} 
However, through this time the fields were never displaced from their minimum, and therefore the dynamics of the flat directions would remain frozen. The universe would be cold, as it was before, and the spatial curvature would remain negative. It is fair to say that, on phenomenological grounds, such a universe is already ruled out. This paves the way for more interesting scenarios, which we discuss next.

\section{B. Negligible Hubble induced corrections}

In this case, the potential is not affected by the false vacuum inflation at all, namely, $|c|,|a| \ll 1$. So long as $V^{\prime \prime}(\phi) \ll H_{\text {false }}^{2}$, the flat direction field $\phi$ makes a quantum jump of length $H_{\text {false }} / 2 \pi$ within each Hubble time. ${ }^{10}$ These jumps superpose in random walk fashion resulting in [19]

$$
\left(\frac{d\left\langle\phi^{2}\right\rangle}{d t}\right)_{\text {fluctuations }}=\frac{H_{\text {false }}^{3}}{4 \pi^{2}} .
$$

On the other hand, the classical slow roll due to the potential leads to

$$
\left(\frac{d\left\langle\phi^{2}\right\rangle}{d t}\right)_{\text {slow roll }}=-\frac{2\left\langle V^{\prime}(\phi) \phi\right\rangle}{3 H_{\text {false }}} .
$$

For a massive scalar field $V(\phi) \sim m_{\phi}^{2} \phi^{2} / 2$, the combined effects yield [19]

$$
\left\langle\phi^{2}\right\rangle=\frac{3 H_{\text {false }}^{4}}{8 \pi^{2} m_{\phi}^{2}}\left[1-\exp \left(-\frac{2 m_{\phi}^{2}}{3 H_{\text {false }}} t\right)\right] .
$$

The maximum field value

$$
\phi_{\text {r.m.s. }}=\sqrt{\frac{3}{8 \pi^{2}}} \frac{H_{\text {false }}^{2}}{m_{\phi}},
$$

at which the slow-roll motion (12) counterbalances the random walk motion (11), is reached for $\Delta t \gg$ $3 H_{\text {false }} / 2 m_{\phi}^{2}$. This amounts to a number

$$
\mathcal{N}_{\text {false }} \gg \frac{3}{2}\left(\frac{H_{\text {false }}}{m_{\phi}}\right)^{2}
$$

of e-foldings of inflation in the false vacuum.

In the absence of Hubble induced corrections, the potential in (10) has a saddle point at

$$
\phi_{0}=\left(\frac{m_{\phi} M_{P}^{n-3}}{\lambda_{n} \sqrt{2 n-2}}\right)^{1 /(n-2)},
$$

where $V^{\prime}\left(\phi_{0}\right)=V^{\prime \prime}\left(\phi_{0}\right)=0$ while $V^{\prime \prime \prime}\left(\phi_{0}\right) \neq 0$, provided that $A^{2}=8(n-1) m_{\phi}^{2} \cdot{ }^{11}$ For $m_{\phi} \sim 100 \mathrm{GeV}-10 \mathrm{TeV}$ and $\lambda_{n} \sim \mathcal{O}(1)$, and for $n=6$, the VEV is $\phi_{0} \sim 10^{14}-10^{15} \mathrm{GeV}$. The suitable flat directions are $L L e$ and $u d d$, which are

\footnotetext{
${ }^{10}$ To be more precise, the quantum fluctuations of $\phi$ have a Gaussian distribution, and the root mean square (r.m.s.) of modes which exit the horizon within one Hubble time is $H_{\text {false }} / 2 \pi$.

${ }^{11}$ This can happen in the gravity mediated case, where $A \sim$ $m_{\phi} \sim m_{3 / 2}$, where $m_{3 / 2} \sim \mathcal{O}(1 \mathrm{TeV})$ is the gravitino mass. The situation is quite different in the case of a gauge mediated SUSY breaking scenario [15].
}

lifted by $n=6$ superpotential terms and also have a nonzero $A$-term as required by the condition for a saddle point $[12,14] .{ }^{12}$

For $\phi<\phi_{0}$ the mass term dominates the flat direction potential. Then quantum fluctuations can push $\phi$ to the vicinity of $\phi_{0}$ if $\phi_{\text {r.m.s. }} \geq \phi_{0} .{ }^{13}$ This, according to (14), requires that

$$
H_{\text {false }} \geq\left(\frac{8 \pi^{2}}{3}\right)^{1 / 4}\left(m_{\phi} \phi_{0}\right)^{1 / 2} \simeq 10^{9} \mathrm{GeV} .
$$

The number of e-foldings needed for this to happen is

$$
\mathcal{N}_{\text {false }} \leq\left(\frac{H_{\text {false }}}{10^{9} \mathrm{GeV}}\right)^{2} 10^{12}
$$

Indeed for $H_{\text {false }} \geq \phi_{0}$ the inherent uncertainty due to quantum fluctuations implies that $\phi>\phi_{0}$ within one Hubble time.

A last stage of MSSM inflation with an expansion rate

$$
H_{\mathrm{MSSM}}=\frac{n-2}{\sqrt{6 n(n-1)}} \frac{m_{\phi} \phi_{0}}{M_{P}} \sim \mathcal{O}(1 \mathrm{GeV})
$$

starts if $V(\phi)$ dominates the energy density of the universe, i.e. $V(\phi)>3 H_{\text {false }}^{2} M_{P}^{2}$. An observationally consistent inflation in the slow-roll regime requires that the displacement from the saddle point satisfy $\left|\phi-\phi_{0}\right|<\Delta \phi$, where $[12,14]$

$$
\Delta \phi=\frac{\phi_{0}^{3}}{4 n(n-1) M_{P}^{2}} \simeq 10^{6} \mathrm{GeV} .
$$

In the landscape, the universe can begin in a false vacuum with arbitrarily large $H_{\text {false }}$ (as long as $H_{\text {false }} \ll M_{P}$ ). Therefore, we generically expect that $\phi$ is quickly pushed to field values $\phi \gg \phi_{0}$. However, $H_{\text {false }}$ slowly decreases as a result of tunneling to vacua with a smaller cosmological constant. ${ }^{14}$ For a massive scalar field with the potential $V(\phi) \sim m_{\phi}^{2} \phi^{2} / 2$, this implies a gradual decrease of $\phi_{\text {r.m.s. }}$; see (14). Since quantum fluctuations can at most push $\phi$ to $\phi_{\text {r.m.s. }}$, this also implies that $\phi$ is slowly decreasing in time.

\footnotetext{
${ }^{12}$ In order to have successful inflation, we require the condition $A^{2}=8(n-1) m_{\phi}^{2}$ to be satisfied to one part in $10^{9}$. Although this requires a fine-tuning, SUSY can allow it to be maintained order by order if $A / m_{\phi}$ acts as an infrared fixed point of the renormalization group flow [14]. Also, as noted in Sec. II A above, this tuning can be explained naturally by the landscape picture. For larger deviations there is a point of inflection with large $V^{\prime}\left(\phi_{0}\right)$ (or a negligible $A$-term discussed in [24]), or a pocket of false minimum [61]. Neither case leads to a slow-roll inflation within the MSSM.

${ }^{13}$ Because of the Gaussian distribution of fluctuations, the probability of having $\phi \gg \phi_{\text {r.m.s. }}$ is exponentially suppressed.

${ }^{14}$ Note that we need to stay in a MSSM-like vacuum all the way until MSSM inflation begins. Given the scarcity of MSSM-like vacua in the landscape, the probability of tunneling from one such vacuum to another is $\sim 10^{-9}$. However, due to eternal inflation in the false vacuum, the physical volume of the universe increases by a factor of $\exp \left(3 H_{\text {false }} / \Gamma\right)$ within a typical time scale for bubble nucleation ( $\Gamma$ is the false vacuum decay rate; see Sec. II B). This easily wins over the suppression factor $10^{-9}$ for $\Gamma<9 H_{\text {false }}$.
} 
Indeed for $H_{\text {false }}<10^{9} \mathrm{GeV}$, we find that $\phi<\phi_{0}$, irrespective of how large $\phi$ initially was. This is the case in the discussion of [7], which is why [7] requires a jump from a large cosmological constant directly to the slow-roll inflationary stage.

Note, however, that the potential becomes very flat around $\phi_{0}$ as a result of the interplay among different terms in (10). In fact, for $\left|\phi-\phi_{0}\right| \ll \phi_{0}$ we have $[12,14]$

$$
V(\phi) \approx V\left(\phi_{0}\right)+\frac{1}{3(n-2)^{2}} \frac{m_{\phi}^{2}}{\phi_{0}}\left(\phi-\phi_{0}\right)^{3}
$$

Once $H_{\text {false }} \sim 10^{9} \mathrm{GeV}, \phi$ reaches this plateau (from above). Within the plateau, $V^{\prime}(\phi)$ becomes increasingly negligible, and so does the classical slow roll; they exactly vanish at $\phi=\phi_{0}$. In consequence, quantum jumps dominate the dynamics and freely move $\phi$ throughout the plateau. Hence the flatness of the potential, which is required for a successful MSSM inflation, guarantees that $\phi$ will remain within the plateau during the landscape evolutionary phase. It is possible to generalize this argument to other models of low-scale inflation; we see that a sufficiently flat inflaton potential can trap inflaton fluctuations in the slow-roll region.

The flat direction eventually dominates the energy density of the universe when $H_{\text {false }}<1 \mathrm{GeV}$; see (19). MSSM inflation then starts in those parts of the universe which obey (20). Having $\phi$ so close to $\phi_{0}$ is possible since the uncertainty due to quantum fluctuations is $\sim 1 \mathrm{GeV}$ at this time.

The false vacuum inflation paves the way for a MSSM inflation inside the nucleated bubble. The modulus which was responsible for the false vacuum inflation continues tunneling to minima with a smaller (eventually the currently observed) cosmological constant inside the MSSM inflating bubble. The modulus will oscillate around the minimum of its potential as the curvature of its potential dominates over the Hubble expansion rate. The fate of oscillations would depend on the coupling of the modulus to the MSSM fields. For a coupling of gravitational strength, oscillations are long-lived. Moreover, the decay will be kinematically forbidden if the decay products are coupled to the flat direction $\phi$ (which has obtained a large $\mathrm{VEV} \simeq \phi_{0}$ ). However, such details are largely irrelevant as the flat direction dominates the energy density of the universe and drives inflation, diluting the energy density in oscillations.

In particular, we note that MSSM inflation has a selfreproducing regime $[12,14]$ because $V^{\prime}(\phi)$ is extremely small and the potential becomes very flat close to $\phi_{0}$. The observable part of our universe can spend an arbitrarily long time in the self-reproducting regime before moving into the standard slow-roll inflation. During this period, the cosmological constant can continue to decay and eventually settle at an observationally acceptable value.
It is already known from [12,14] that the flat direction generates a large number of e-foldings during slow-roll inflation, i.e. $\mathcal{N}_{e} \sim 10^{3}$, and during this phase the amplitude of the scalar perturbations and the spectral index are given by $[12,14]$

$$
\begin{aligned}
\delta_{H} & \equiv \frac{1}{5 \pi} \frac{H_{\mathrm{MSSM}}^{2}}{\dot{\phi}} \\
& \simeq \frac{1}{5 \pi} \sqrt{\frac{1}{6} n(n-1)(n-2)\left(\frac{m_{\phi} M_{P}}{\phi_{0}^{2}}\right) \mathcal{N}_{\mathrm{COBE}}^{2}}
\end{aligned}
$$

$$
n_{s}=1+2 \eta-6 \epsilon \simeq 1-\frac{4}{\mathcal{N}_{\mathrm{COBE}}} .
$$

Here $\mathcal{N}_{\mathrm{COBE}} \sim 50$ is the relevant number of e-foldings of inflation required to explain the current observations. For $m_{\phi} \sim 100 \mathrm{GeV}-10 \mathrm{TeV}, \quad n=6, \phi_{0} \sim 10^{14}-10^{15} \mathrm{GeV}$, we obtain the correct amplitude of density perturbations and the scalar spectral index $\left(n_{s}=0.92\right.$, within $2 \sigma$ of the current WMAP results). ${ }^{15}$ Actually, the value of $\mathcal{N}_{\text {COBE }}$ depends on the thermal history of the universe. In our case, the precise thermal history is known because the MSSM flat direction has SM couplings, and we know the maximum (see below) and the reheat temperatures [21,62-64].

Slow-roll inflation ends when the slow-roll conditions are violated. Eventually, the flat direction starts oscillating around the origin with a frequency $m_{\phi} \sim 1 \mathrm{TeV}$. The flat direction condensate decays via instant preheating and creates a relativistic bath of MSSM quanta with a maximum temperature $[12,14]$

$$
T_{\max } \sim\left[V\left(\phi_{0}\right)\right]^{1 / 4} \sim 10^{9} \mathrm{GeV} .
$$

The temperature is sufficiently high to trigger electroweak baryogenesis within the MSSM and to provide the sufficient thermal condition for production of cold dark matter, i.e. the lightest supersymmetric particle (LSP).

Before we conclude this discussion, let us remind the readers that obtaining the flat direction VEV, $\phi_{0}$, depends on the false vacuum inflation, which requires $H_{\text {false }} \geq$ $10^{9} \mathrm{GeV}$ at some time [see (17)]. This condition, although very probable in the landscape picture, need not always be satisfied. Then the quantum fluctuations would not be large enough to push the flat direction to the vicinity of $\phi_{0}$ as required for a final stage of MSSM inflation. This would therefore ruin the inflationary and phenomenological predictions. This can be avoided if the flat direction is trapped in a false minimum which evolves with time, as discussed below.

\footnotetext{
${ }^{15}$ The above results, (22) and (23), are strictly true in the saddle point limit. A small deviation from the saddle point condition predicts a spectral tilt $0.92 \leq n_{s} \leq 1.0$ with an observable range and negligible running and tensor perturbations $[25,26]$.
} 


\section{Negative Hubble induced corrections}

The case with $c \sim-\mathcal{O}(1)$ may arise naturally for a nonminimal Kähler potential [22,23]. For $H_{\text {false }} \gg m_{\phi}$ the potential in (10) becomes tachyonic, and its true minimum is located at

$$
\phi_{\min } \simeq\left(\frac{\sqrt{|c|}}{\lambda_{n} \sqrt{2 n-2}} H_{\text {false }} M_{P}^{n-3}\right)^{1 /(n-2)} .
$$

Note that $\phi_{\min }$ is initially larger than $\phi_{0}$; see (16).

The curvature of the potential at the minimum is $V^{\prime \prime}\left(\phi_{\min }\right)=(4 n-7)|c| H_{\text {false }}^{2} \gg H_{\text {false }}^{2}$. This implies that $\phi$ is driven away from the origin, due to quantum fluctuations, and quickly settles down at $\phi_{\min }$. The MSSM flat direction is trapped inside the minimum, held due to false vacuum inflation, and gradually tracks the instantaneous value of $\phi_{\min }$ as $H_{\text {false }}$ decreases. ${ }^{16}$

The minima at $\phi=0$ and $\phi=\phi_{\min }$ become degenerate when $H_{\text {false }} \sim m_{\phi}$. For $H_{\text {false }} \ll m_{\phi}$ the true minimum is at $\phi=0$ and the one at $\phi_{\min }$ will be false. The Hubble induced corrections are subdominant in this case. We then find [14]

$$
\begin{aligned}
& \phi_{\min } \simeq \phi_{0}\left(1+\frac{1}{n-2} \frac{\sqrt{|c|} H_{\text {false }}}{m_{\phi}}\right), \\
& V^{\prime \prime}\left(\phi_{\min }\right) \simeq 2(n-2) \sqrt{|c|} H_{\text {false }} m_{\phi} .
\end{aligned}
$$

The $\phi$ field can track down the instantaneous value of $\phi_{\text {min }}$ provided that $\sqrt{V^{\prime \prime}\left(\phi_{\min }\right)}$ is greater than the Hubble expansion rate. In fact, this is the case so long as $H_{\text {false }}>$ $H_{\mathrm{MSSM}} \sim 1 \mathrm{GeV}$; see (27). Once $H_{\text {false }} \simeq H_{\mathrm{MSSM}}$, the flat direction potential dominates the energy density of the universe, and MSSM inflation begins at a Hubble expansion rate $H_{\mathrm{MSSM}}$. In the meantime, landscape tunneling to vacua with a smaller cosmological constant continues, and the location of the false minimum $\phi_{\min }$ continuously changes. ${ }^{17}$ Eventually $V^{\prime \prime}\left(\phi_{\text {min }}\right)<H_{\text {MSSM }}^{2}$ when

$$
H_{\text {false }} \simeq \frac{1}{2(n-2)} \frac{H_{\mathrm{MSSM}}^{2}}{m_{\phi}},
$$

at which time

$$
\phi_{\min }-\phi_{0} \simeq \frac{\phi_{0}}{2(n-2)^{2}}\left(\frac{H_{\mathrm{MSSM}}}{m_{\phi}}\right)^{2} .
$$

It turns out from (19) and (20) that $\phi_{\min }-\phi_{0} \ll \Delta \phi$. Therefore $\phi$ is already inside the interval required for a successful MSSM inflation. At this point the Hubble in-

\footnotetext{
${ }^{16}$ Here we are assuming that the difference between the energy densities of the two false vacua is small compared to their average energy density.

${ }^{17}$ Note that tunnelings do not affect the Hubble expansion rate anymore since the flat direction dominates the energy density of the universe now.
}

duced corrections become largely unimportant, and all the successes of MSSM inflation are retained, as discussed in the previous subsection. The fate of the string moduli inside the MSSM bubble remains the same as in the previous subsection. In particular, it does not matter whether the universe tunnels right away to the currently observed value of $\Lambda$ or not. Inflation dilutes any excitations of the modulus oscillations during inflation. Our Hubble patch reheats when the MSSM flat direction rolls down to its minimum and starts creating MSSM quanta as discussed in the previous subsection.

Note that all that is needed for the success of this scenario is to start in a false vacuum in the landscape where $H_{\text {false }}>m_{\phi} \sim 1 \mathrm{TeV}$. This ensures that the flat direction will roll away from the origin and settle at $\phi_{\min }$, which is the true minimum of $V(\phi)$ at that time. It will then track $\phi_{\min }$ as $H_{\text {false }}$ slowly decreases, and will eventually land inside the appropriate interval around $\phi_{0}$. This is a much milder condition than that in the case of negligible Hubble induced corrections $H_{\text {false }} \geq 10^{9} \mathrm{GeV}$ (see the previous subsection).

One comment is in order. This scenario has some similarities to the Affleck-Dine baryogenesis with negative Hubble induced corrections [22,23]. It can be seen from (10) that the equation of motion of the flat direction has a fixed point so long as Hubble induced corrections are dominant. The flat direction tracks the fixed point in a radiation or matter dominated universe. However, a nonadiabatic change in the potential occurs when $H \sim m_{\phi}$, and the flat direction starts oscillating around the origin. However, in our case the universe is in a de Sitter phase with a slowly varying $H_{\text {false }}$, and the flat direction tracks the false minimum of its potential until it dominates the universe. As explained, this is necessary to have a successful MSSM inflation.

\section{CONCLUSION AND DISCUSSION}

In this paper, we have developed a modified version of "chain inflation" which has a graceful exit due to MSSM inflation. Multiple stages of false vacuum inflation relax the cosmological constant and provide the appropriate initial conditions for inflation driven by the MSSM flat direction [12,14] (as follows from an improved version of arguments from [7]). This last stage inflates the universe to its current size and generates acceptable density perturbations which match the current observations. Furthermore, MSSM inflation will dilute any relics of string theory and has a negligible gravity wave background, so it has a clear prediction of standard inflationary cosmic microwave background (CMB) perturbations with no gravity waves, which is relevant for upcoming experiments. Without the initial stages of high energy false vacuum inflation, it would be very difficult to explain the initial conditions for MSSM inflation, and without the latter there would be no graceful exit from the false vacuum inflation. We find 
the complementarity of these two types of inflation to be intriguing.

Moreover, MSSM inflation produces a self-reproduction regime and many e-foldings of slow-roll inflation, which is important in solving the cosmological constant problem via tunneling. As discussed in [50,51], self-reproduction allows the cosmological constant to settle down to its present value long before the final 60 e-foldings of inflation, which allows for a standard CMB perturbation spectrum. Also, [51] points out that $\mathrm{TeV}$ scale eternal inflation can fit observational constraints, even after tunneling events in the context of string and $M$ theory, which is another point of interest for MSSM inflation. In addition, self-reproduction is a virtue which is difficult to find among inflationary mechanisms at scales below the string scale. For instance, brane-antibrane inflation (for a recent review see [65]) and multiple-axion-driven [66] assisted inflation $[67,68]$ fail to provide large e-foldings of inflation without fine-tuning of the Lagrangian. On the other hand, "roulette inflation" [69] and "racetrack inflation" [70,71] can give rise to self-reproduction, and it would be interesting to study either of these models as a graceful exit from false vacuum inflation on the landscape and how false vacuum inflation can provide the proper initial conditions for those types of inflation.

\section{ACKNOWLEDGMENTS}

We are thankful to Raphael Bousso, Robert Brandenberger, Kari Enqvist, and Juan Garcia-Bellido for discussion at various stages of this work. The work of R. A. is supported by Perimeter Institute for Theoretical Physics. Research at Perimeter Institute is supported in part by the Government of Canada through NSERC and by the province of Ontario through MRI. A. R. F. is partly supported by IPP/PI and partly by le Fonds Nature et Technologies du Québec. A. M. is partly supported by the European Union through Marie Curie Research and Training Network "UNIVERSENET" (MRTN-CT-035863).

\section{APPENDIX: TUNNELING FORMULAS}

For completeness, we list here the instanton bubble radius and effective tension:

$$
\frac{1}{r^{2}}=\frac{\tau^{2}}{M_{P}^{4}}\left[\frac{1}{16}+\frac{1}{6}\left(\lambda_{+}+\lambda_{-}\right)+\frac{1}{9}\left(\lambda_{+}-\lambda_{-}\right)^{2}\right]
$$

and

$$
\begin{aligned}
\tau_{e}= & \tau\left\{1+\frac{6}{\lambda_{+} \lambda_{-}}\left[\lambda_{+}\left|\frac{1}{4}+\frac{1}{3}\left(\lambda_{+}-\lambda_{-}\right)\right|^{3}+\lambda_{-} \mid \frac{1}{4}\right.\right. \\
& +\left.\frac{1}{3}\left(\lambda_{-}-\lambda_{+}\right)\right|^{3}+\left(\lambda_{-}-\lambda_{+}\right)\left(\frac{1}{16}+\frac{1}{6}\left(\lambda_{+}+\lambda_{-}\right)\right. \\
& \left.\left.\left.+\frac{1}{9}\left(\lambda_{+}-\lambda_{-}\right)^{2}\right)^{3 / 2}\right]\right\} .
\end{aligned}
$$

We will also give the details of the decay rate calculation for the two examples of chain inflation listed in Sec. II B. The first case, ignoring warping, was given in [10], and we will recap here. For a perturbative large volume compactification with many fluxes, the cosmological constant is given by (1) with the constants $c_{i} \ll 1$. We also take an isotropic compactification, so all the $c_{i}$ are approximately equal. Further, the brane charge $q$ and Planck mass (we follow here the conventions of [10] except that we take $q \rightarrow M_{P}^{2} q$ ) become

$$
q \approx c, \quad M_{P}^{2} \approx 1 / c \alpha^{\prime}
$$

The instanton bubble tension is then $\tau \sim M_{P} / \alpha^{\prime}$, so we find $\lambda_{+} \sim M_{P}^{2} \alpha^{\prime} c n^{2} \sim n^{2} \gg 1$. Finally, the Euclidean action of the instanton is

$$
\Delta S_{E} \approx \frac{\tau}{\Lambda_{+}^{3 / 2}} \approx \frac{1}{c n^{3}}
$$

As long as $c^{-1 / 3} \ll n \ll c^{-1}, \Delta S_{E} \ll 1$ and $\Lambda_{+} \ll M_{P}^{2}$.

In the second case, we look at a compactification with a similar 5-brane instanton decay localized in a warped throat. This decay has been studied in [42,49]. A perturbative $g_{s} \sim 1 / 10 \lesssim 1$ large volume compactification $e^{u} \sim$ $10 \gtrsim 1$ [with $e^{u}$ the linear scale of the (unwarped) compactification manifold] has a Newton constant

$$
\frac{1}{2 \kappa_{4}^{2}} \approx \frac{1}{2 \pi \alpha^{\prime}}
$$

(Here, we follow the conventions of [42], in which the VEVs of $u$ and $g_{s}$ are scaled out of the Einstein frame metric.) It is possible to see that the instanton bubble tension is dominated by a term

$$
\tau \approx \frac{1}{g_{s}^{1 / 2} e^{6 u}} \frac{|z|}{\alpha^{13 / 2}},
$$

where $z$ is the complex structure modulus of the warped throat. This modulus is related to the minimum warp factor of the throat in such a way that

$$
\tau \approx \ell_{s w}^{-2}\left(g_{s} M\right)^{3 / 2} g_{s}^{-5 / 4} e^{-9 u} \approx 10^{-2} \ell_{s w}^{-2},
$$

where $M$ is a flux quantum number, $g_{s} M \sim 10 \gtrsim 1$, and $\ell_{s w}$ is the warped string length at the bottom of the throat.

Now let us imagine that this decay does not annihilate most of the cosmological constant. Then [20] argues that $\ell_{s w}^{-2} \sim \Lambda$. This immediately implies that $\lambda_{+} \gtrsim M_{P}^{4} / \Lambda^{2} \gg$ 1 and $\Delta S_{E} \sim 1$. (We know that $\lambda_{-} \sim \lambda_{+}$because the energy density localized in the warped region is $1 / \ell_{s w}^{4} \sim$ $\Lambda^{2} \ll M_{P}^{2} \Lambda$.) 
[1] M. R. Douglas, C.R. Physique 5, 965 (2004).

[2] J. Kumar, Int. J. Mod. Phys. A 21, 3441 (2006).

[3] M. R. Douglas and S. Kachru, Rev. Mod. Phys. 79, 733 (2007).

[4] A. H. Guth, Phys. Rev. D 23, 347 (1981).

[5] C. P. Burgess, R. Easther, A. Mazumdar, D. F. Mota, and T. Multamaki, J. High Energy Phys. 05 (2005) 067.

[6] L. F. Abbott, Phys. Lett. 150B, 427 (1985).

[7] R. Bousso and J. Polchinski, J. High Energy Phys. 06 (2000) 006.

[8] B. Freivogel and L. Susskind, Phys. Rev. D 70, 126007 (2004).

[9] S. H. Henry Tye, arXiv:hep-th/0611148.

[10] K. Freese, J. T. Liu, and D. Spolyar, arXiv:hep-th/ 0612056.

[11] D. N. Spergel et al. (WMAP), arXiv:astro-ph/0603449.

[12] R. Allahverdi, K. Enqvist, J. Garcia-Bellido, and A. Mazumdar, Phys. Rev. Lett. 97, 191304 (2006).

[13] R. Allahverdi, A. Kusenko, and A. Mazumdar, arXiv:hep$\mathrm{ph} / 0608138$.

[14] R. Allahverdi, K. Enqvist, J. Garcia-Bellido, A. Jokinen, and A. Mazumdar, arXiv:hep-ph/0610134.

[15] R. Allahverdi, A. Jokinen, and A. Mazumdar, arXiv:hep$\mathrm{ph} / 0610243$.

[16] K. Enqvist and A. Mazumdar, Phys. Rep. 380, 99 (2003).

[17] M. Dine and A. Kusenko, Rev. Mod. Phys. 76, 1 (2004).

[18] G. Jungman, M. Kamionkowski, and K. Griest, Phys. Rep. 267, 195 (1996).

[19] A. D. Linde, arXiv:hep-th/0503203.

[20] A. R. Frey, A. Mazumdar, and R. Myers, Phys. Rev. D 73, 026003 (2006).

[21] R. Allahverdi and A. Mazumdar, arXiv:hep-ph/0603244.

[22] M. Dine, L. Randall, and S. D. Thomas, Phys. Rev. Lett. 75, 398 (1995).

[23] M. Dine, L. Randall, and S. D. Thomas, Nucl. Phys. B458, 291 (1996).

[24] A. Jokinen and A. Mazumdar, Phys. Lett. B 597, 222 (2004).

[25] R. Allahverdi and A. Mazumdar, arXiv:hep-ph/0610069.

[26] J. C. Bueno Sanchez, K. Dimopoulos, and D. H. Lyth, J. Cosmol. Astropart. Phys. 01 (2007) 015.

[27] R. Allahverdi and A. Mazumdar, arXiv:hep-ph/0610069.

[28] R. Allahverdi, K. Enqvist, J. Garcia-Bellido, A. Jokinen, and A. Mazumdar, arXiv:hep-ph/0610134.

[29] D. H. Lyth, J. Cosmol. Astropart. Phys. 04 (2007) 006.

[30] G. R. Dvali, arXiv:hep-ph/9503259.

[31] M. Dine, L. Randall, and S. D. Thomas, Phys. Rev. Lett. 75, 398 (1995).

[32] M. Dine, L. Randall, and S. D. Thomas, Nucl. Phys. B458, 291 (1996).

[33] R. Blumenhagen, F. Gmeiner, G. Honecker, D. Lust, and T. Weigand, Nucl. Phys. B713, 83 (2005).

[34] F. Gmeiner, R. Blumenhagen, G. Honecker, D. Lust, and T. Weigand, J. High Energy Phys. 01 (2006) 004.

[35] F. Gmeiner, Fortschr. Phys. 54, 391 (2006).

[36] M. R. Douglas and W. Taylor, J. High Energy Phys. 01 (2007) 031

[37] S. Kachru, R. Kallosh, A. Linde, and S. P. Trivedi, Phys. Rev. D 68, 046005 (2003).

[38] N. Arkani-Hamed, S. Dimopoulos, and S. Kachru, arXiv:hep-th/0501082.
[39] T. Banks, arXiv:hep-th/0211160.

[40] T. Clifton, A. Linde, and N. Sivanandam, J. High Energy Phys. 02 (2007) 024.

[41] J. Garriga, Phys. Rev. D 49, 6327 (1994).

[42] A. R. Frey, M. Lippert, and B. Williams, Phys. Rev. D 68, 046008 (2003).

[43] S. R. Coleman and F. De Luccia, Phys. Rev. D 21, 3305 (1980).

[44] J. D. Brown and C. Teitelboim, Phys. Lett. B 195, 177 (1987).

[45] J. D. Brown and C. Teitelboim, Nucl. Phys. B297, 787 (1988).

[46] K. Freese and D. Spolyar, J. Cosmol. Astropart. Phys. 07 (2005) 007.

[47] K. Freese, J. T. Liu, and D. Spolyar, Phys. Rev. D 72, 123521 (2005).

[48] U.H. Danielsson, N. Johansson, and M. Larfors, J. High Energy Phys. 03 (2007) 080.

[49] S. Kachru, J. Pearson, and H. L. Verlinde, J. High Energy Phys. 06 (2002) 021.

[50] R. Bousso and A. Chamblin, Phys. Rev. D 59, 063504 (1999).

[51] J. Garriga and A. Vilenkin, Phys. Rev. D 64, 023517 (2001).

[52] S. W. Hawking and I. G. Moss, Phys. Lett. 110B, 35 (1982).

[53] P. Batra and M. Kleban, arXiv:hep-th/0612083.

[54] E. J. Weinberg, arXiv:hep-th/0612146 [Phys. Rev. Lett. (to be published)].

[55] A. Ceresole, G. Dall'Agata, A. Giryavets, R. Kallosh, and A. Linde, Phys. Rev. D 74, 086010 (2006).

[56] A. Linde, J. Cosmol. Astropart. Phys. 01 (2007) 022.

[57] R. Kallosh, A. D. Linde, D. A. Linde, and L. Susskind, Phys. Rev. D 52, 912 (1995).

[58] T. Gherghetta, C. F. Kolda, and S. P. Martin, Nucl. Phys. B468, 37 (1996).

[59] M. K. Gaillard, H. Murayama, and K. A. Olive, Phys. Lett. B 355, 71 (1995).

[60] R. Allahverdi, M. Drees, and A. Mazumdar, Phys. Rev. D 65, 065010 (2002).

[61] R. Allahverdi, K. Enqvist, A. Jokinen, and A. Mazumdar, J. Cosmol. Astropart. Phys. 10 (2006) 007.

[62] R. Allahverdi and A. Mazumdar, arXiv:hep-ph/0608296.

[63] R. Allahverdi and A. Mazumdar, arXiv:hep-ph/0505050.

[64] R. Allahverdi and A. Mazumdar, J. Cosmol. Astropart. Phys. 10 (2006) 008.

[65] J. M. Cline, arXiv:hep-th/0612129.

[66] S. Dimopoulos, S. Kachru, J. McGreevy, and J. G. Wacker, arXiv:hep-th/0507205.

[67] A. R. Liddle, A. Mazumdar, and F. E. Schunck, Phys. Rev. D 58, 061301 (1998).

[68] E. J. Copeland, A. Mazumdar, and N. J. Nunes, Phys. Rev. D 60, 083506 (1999).

[69] J.R. Bond, L. Kofman, S. Prokushkin, and P. M. Vaudrevange, arXiv:hep-th/0612197 [Phys. Rev. D (to be published)].

[70] J. J. Blanco-Pillado et al., J. High Energy Phys. 11 (2004) 063.

[71] J. J. Blanco-Pillado et al., J. High Energy Phys. 09 (2006) 002. 